\title{
Direct Determination of Spatial Alignments of Metal Nanoparticles in Bicontinuous Polymer Matrix Studied by Transmission Electron Microtomography
}

\author{
H. Jinnai, * T. Kaneko, * H. Nishioka, ** Y. Nishikawa, ${ }^{*}$ H. Hasegawa, *** T. Nishi****
}

* Department of Polymer Science and Engineering, Kyoto Institute of Technology, Matsugasaki, Sakyo-ku, Kyoto 606-8585, Japan

** Joint Research Center for Project on Nanostructure Polymeric Materials, Japan Chemical Innovation Institute (JCII), Kyoto Institute of Technology, Matsugasaki, Sakyo-ku, Kyoto 606-8585, Japan

*** Department of Polymer Chemistry, Graduate School of Engineering, Kyoto University, Kyoto, 615-8510, Japan

**** Department of Organic and Polymeric Materials, School of Science and Engineering, Tokyo Institute of Technology, 2-12-1, Ohokayama, Meguro-ku, Tokyo, 152-8552, Japan

Fine metal particles with a diameter of the order of nanometers (designated hereafter as "nanoparticles") are of current interest for applications such as metal catalysts, optical devices, etc [1]. In order to realize such functionalities, control of spatial distribution as well as stabilization of the nanoparticles inside the materials are important. Such spatial distribution of the nanoparticles may be achieved by use of surfactants [2] or polymers [1,3] as the substrates in order to stabilize the particles on their surfaces.

A block copolymer, poly(2-vinylpyridine-block-isoprene) (P2VP-b-PI), forms Gyroid (G) nanostructure with $I a \overline{3} d$ cubic symmetry. In the present study, the G nanostructure was used as the substrate. The P2VP network phase was first crosslinked with diiodobutane. Subsequently, the PI matrix was degraded by ozonolysis. Thus, the freestanding Gyroid network texture was created, in which Pd nanoparticles were formed presumably on the surface of the P2VP via reduction of $\mathrm{Pd}^{2+}$ ions with 1-propanol as reductant. Although the Pd particles seemed to be fixed on the P2VP surface and were uniformly distributed in the G texture, their spatial distribution has to be determined in 3D. Note that this system $(\mathrm{P} 2 \mathrm{VP} / \mathrm{Pd})$ is a model of high-performance catalyst since the polymeric nanoporous material used here has substantially large surface area (and hence large number of Pd particles) for a given volume. The ultra-thin section of the P2VP film with Pd nanoparticles inside was prepared by a microtome (Reichelt Ultracut UCT) at room temperature. TEMT observation was performed on JEM-2200FS (JEOL Ltd., Japan) operated at $200 \mathrm{KV}$. The energy filter equipped in the TEM was used for eliminating the chromatic aberration to obtain the "zero-loss" images.

Figures 1(a) and 1(b) display, respectively, orthogonal cross-sectional views of the 3D reconstructed data of the P2VP/Pd sample: The figure consists of three parts, i.e. the lateral plane (x-y plane), $\mathrm{x}-\mathrm{z}$ and $y-z$ planes. Note that the weighted back-projection was performed in the $x-z$ plane. The highly periodic and regular G morphology (shown by gray area in the figure) was clearly seen in all crosssections. Small dots in the cross-sections were identified as Pd nanoparticles by the electron energy loss spectroscopy. The thickness of the ultra-thin section was estimated to be ca. $220 \mathrm{~nm}$ from $\mathrm{x}-\mathrm{z}$ plane. Interface of the P2VP phase was determined by binarizing the $\mathrm{x}-\mathrm{y}$ slices with a threshold. Similarly, larger threshold value than that used for detecting P2VP was employed to detect the position of the $\mathrm{Pd}$ nanoparticles. 
A 3D image of the 3D reconstruction is shown in Fig. 2, in which Pd nanoparticles appeared to "sit" on the P2VP interface or embedded in the P2VP matrix [see also Fig. 2(b)]. The particle density evaluated from the 3D reconstruction was $490\left(\mu \mathrm{m}^{-3}\right)$ and the volume fraction of the P2VP was 0.44. Besides such basic structural parameters, histograms of diameter of Pd nanoparticles [Fig. 3(a)] and distance between the center of the particles and P2VP interface [Fig. 3(b)] were obtained by image analysis. From the histograms shown in Fig. 3(a), the average diameter and standard deviation of the $\mathrm{Pd}$ particles were, respectively, $4.2 \mathrm{~nm}$ and 0.7 . Since the distance between the particle and interface agreed with the diameter of the particles, it was qualitatively confirmed that the $\mathrm{Pd}$ nanoparticles were halfway embedded in the polymer substrate. Note the distance is particularly important since whether or not the metal particles are completely embedded or just sit on the substrate strongly affects the performance of the material as a catalyst. It would also be interesting to study a relation between the surface curvature and local density of the nanoparticles, if any, which would only be investigated by TEMT.

\section{References}

[1] H.Tamai et al., J. Appl. Polym. Sci.. 56 (1995) 441.

[2] J.P. Spatz et al., Chem. Eur. J.. 2 (1996) 1552.

[3] K. Tsutsumi et al., Langmuir, 15 (1999) 5200.

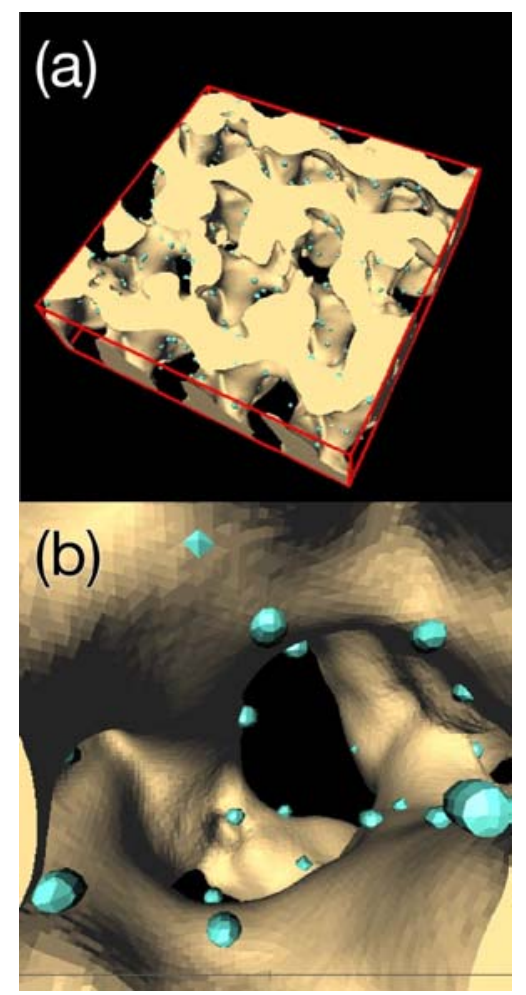

Fig. 2 (a) 3D reconstruction of P2VP/Pd system. Box size is $600 \mathrm{~nm} \times 600 \mathrm{~nm} \times 170 \mathrm{~nm}$. (b) Close-up view of $3 \mathrm{D}$ reconstructed image. Brown solid body and blue dots represent, respectively, P2VP phase and Pd nanoparticles.

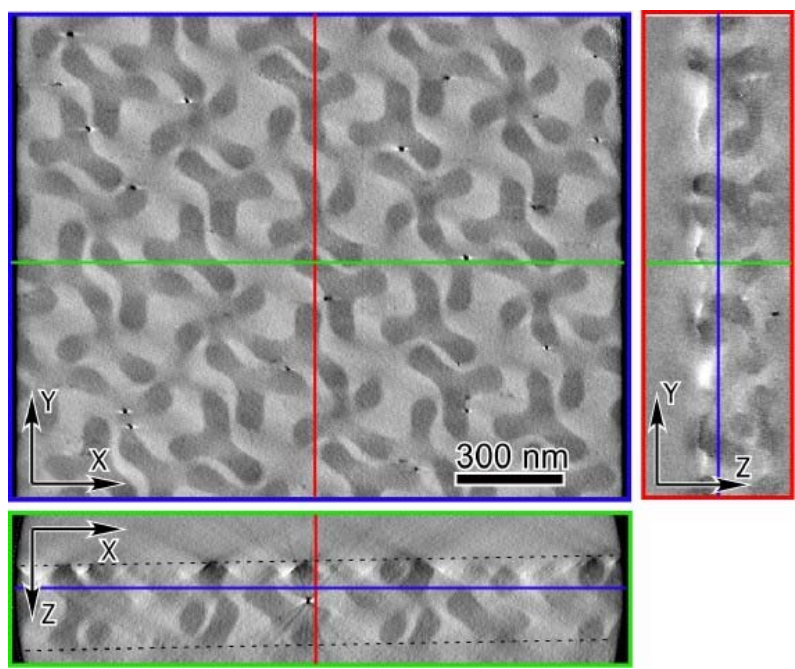

Fig. 1 Three orthogonal cross-sections of 3D reconstruction in $\mathrm{P} 2 \mathrm{VP} / \mathrm{Pd}$ system. Dots in the cross-sections are $\mathrm{Pd}$ nanonarticles Dashed line in $\mathrm{x}-7$. nlane renresents ultra-thin
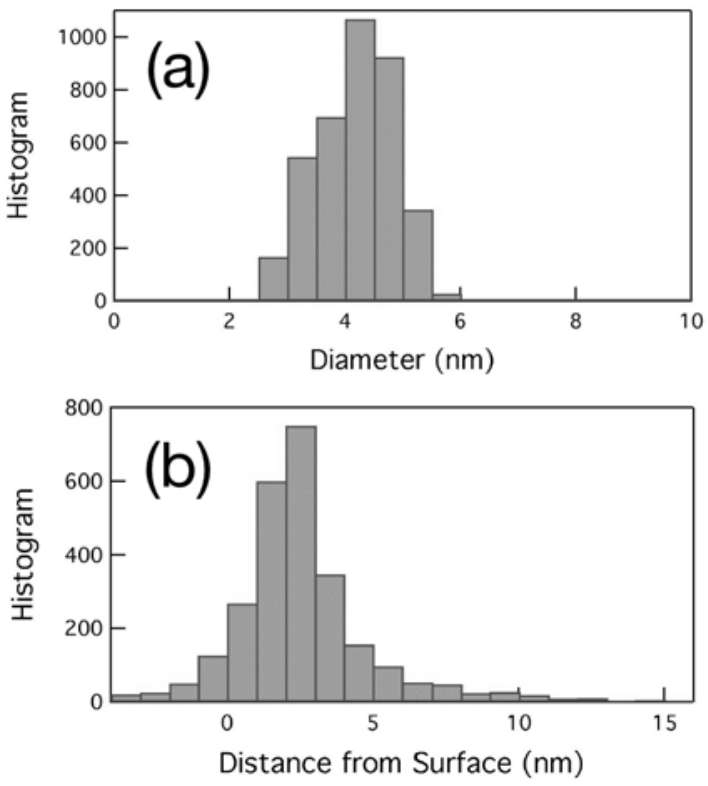

Fig. 3 Histograms of (a) diameter and (b) distance from P2VP surface of Pd nanoparticles. 